\title{
Defined Notification Message
}

National Cancer Institute

\section{Source}

National Cancer Institute. Defined Notification Message. NCI Thesaurus. Code $C 93773$.

The text that is to be included in the notification. 